EPJ Web of Conferences 41, 05008 (2013)

DOI: $10.1051 /$ epjconf/20134105008

(C) Owned by the authors, published by EDP Sciences, 2013

\title{
The interplay of different relaxation channels in the excited state dynamics of photoinitiators
}

\author{
T. J. A. Wolf ${ }^{1,2, a}$, J. Fischer ${ }^{2,3}$, D. Voll ${ }^{2,5}$, M. Wegener ${ }^{2,3,4}$, C. Barner-Kowollik ${ }^{2,5}$, and A.-N. \\ Unterreiner $^{1,2}$ \\ ${ }^{1}$ Institut für Physikalische Chemie, Karlsruhe Institute of Technology (KIT), 76128 Karlsruhe, Ger- \\ many \\ 2 DFG Center for Functional Nanostructures (CFN), KIT \\ 3 Institut für Angewandte Physik, KIT \\ 4 Institut für Nanotechnologie, KIT \\ 5 Preparative Macromolecular Chemistry, Institut für Technische Chemie und Polymerchemie, KIT
}

\begin{abstract}
Different photoinitiators are investigated by femtosecond transient absorption spectroscopy and DFT calculations. Their initiation properties are critically governed by competition between intersystem crossing, fluorescence and internal conversion partly taking place on comparable time scales. It is thereby shown that the $\mathrm{S}^{*}$ lifetime cannot serve as a measure for the photoinitiation efficiency.
\end{abstract}

\section{Introduction and experimental}

Photoinduced radical polymerization is a method with increasing importance due to promising applications including photocuring [1] and lithography [2-4]. A critical process is thereby the photoinduced polymerization initiation by triplet photoinitiators. After photoexcitation into a singlet state $\left(\mathrm{S}^{*}\right)$ they perform intersystem crossing (ISC) into the triplet manifold, from where polymerization initiation can take place [6]. In earlier studies we found strong hints for the overall efficiency of initiation being governed by the quantum efficiency of ISC within the photoinitiator [5,6]. The aim of the present study is to determine, whether there is a direct connection between $\mathrm{S}^{*}$ lifetime and the efficiency of ISC, i.e. the overall photoinitiation efficiency. Such a connection could provide a comparably easy-accessible measure for the suitability of a triplet photoinitiator. For this purpose we comparatively investigated two "classical" photoinitiators, which are used e.g. for pulsed laser polymerization (PLP) [5] and two photoinitiators specially suitable for stimulated emission depletion (STED) lithography [4,7-9].

DETC and ITX were dissolved in ethanol, Bz, TMB and Me in methanol. All transient absorption (TA) experiments were conducted with the same laser system $[6,8]$ using the output of a noncollinear optical parametric amplifier (NOPA), which was upconverted by a portion of the laser fundamental realizing pump pulses at $351 \mathrm{~nm}$ or by the second harmonic of the laser fundamental resulting in pump pulses at $388 \mathrm{~nm}$ and the visible output of a second NOPA as probe pulses. For quantum chemical calculation the TURBOMOLE V6.3 program package [11] was employed. Geometry optimizations were conducted with BP86/def2-SV(P), excitations were calculated with TDDFT/B3LYP/aug-cc-pVDZ.

\section{Results and discussion}

The four photoinitiators discussed in this section are ordered with respect to their $\mathrm{S}^{*}$ lifetime. The experimental absorption spectrum of Mesitil, the system with the shortest $\mathrm{S}^{*}$ lifetime, is shown in

\footnotetext{
a e-mail: thomas.wolf@kit.edu
}

This is an Open Access article distributed under the terms of the Creative Commons Attribution License 2.0, which permits unrestricted use, distribution, and reproduction in any medium, provided the original work is properly cited. 

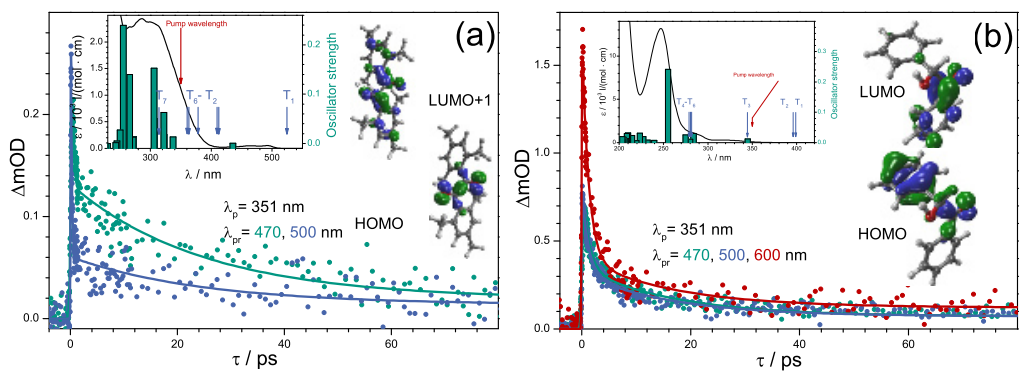

Fig. 1. Transient absorption traces (dots) of (a) Mesitil and (b) Benzoin with global fit analysis. Inserted are the experimental absorption spectra in methanol together with calculated transitions to excited singlet and triplet states. Additionally the MOs, which are relevant for description of the state excited by $\lambda_{p}$, are depicted.

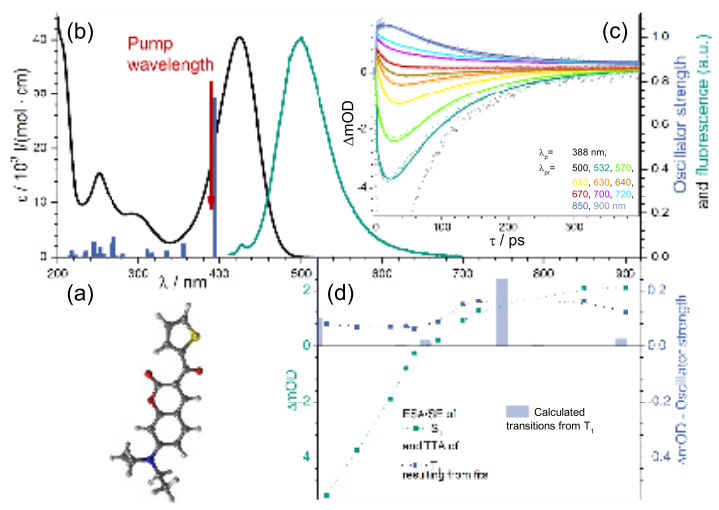

Fig. 2. (a) Structure of DETC, (b) absorption and fluorescence spectra and calculated singlet transitions, (c) transient absorption traces at several $\lambda_{p r}$ with global fit analysis, (d) amplitudes of excited state absorption/stimulated emission (SE/ESA) and triplet-triplet absorption (TTA) together with calculated triplet transitions from $\mathrm{T}_{1}$.

Fig. 1 (a) together with calculated singlet and triplet excitations and TA traces. Excitation of the red wing of the second absorption band can be assigned to population of $\mathrm{S}_{2}$, which corresponds to a LUMO $+1 \leftarrow$ HOMO excitation (see the visualization of the MOs in Fig. 1 (a)). The initial decay of TA traces can be assigned to the $S_{2}$ lifetime of 0.2 ps [6]. Mesitil is observed to exhibit a considerably lower photoinitiation efficiency than the structurally similar Benzoin (see below) [5]. ISC therefore seems to be only a minor exit channel for $\mathrm{S}_{2}$ population. As can be estimated from the highly delocalized $\pi$-system and the calculated singlet excitations, the density of states and therefore the probability for additional highly efficient deactivation channels via internal conversion (IC) is very high. Such processes probably account for the majority of $\mathrm{S}_{2}$ population.

The main difference between the otherwise similar systems Benzoin and Mesitil is the $\mathrm{sp}^{3}$ carbon atom between the aromatic rings in Benzoin isolating them efficiently from each other. This can be seen in the nicely separated electron density of the HOMO and LUMO (see Fig. 1 (b)). For the $\mathrm{S}_{1}$ state, which was excited in the experiments (see experimental and calculated spectrum in Fig. 1 (b)) corresponding to a LUMO $\leftarrow$ HOMO transition, a triplet state very close in energy therefore can be assumed, which is also retrieved by the calculations. Since ISC is crucially sensitive on the singlettriplet energy gap, it is therefore very fast. Thus, the $\mathrm{S}_{1}$ lifetime of $1.2 \mathrm{ps}$, which is observed in the TA traces (see Fig. 1 (b)), is governed by ISC [6]. The comparison of Mesitil and Benzoin therefore yields a growth in ISC efficiency with growing $\mathrm{S}^{*}$ lifetime.

As can be seen from the absorption/fluorescence spectra in Fig. 2 (b) and a considerable fluorescence quantum yield (2.5\%) [9], the $\mathrm{S}_{1}$ lifetime of DETC (structure: Fig. 2 (a)) has to be significantly larger than in the first two cases. From the TA traces in Fig. 2 (c) a value of 99 ps could be extracted. Thus, the time scale of ISC, which accounts for the major part of the population, has to be also substantially larger than in the first two examples. The spectrum of DETC shows typical properties of a 


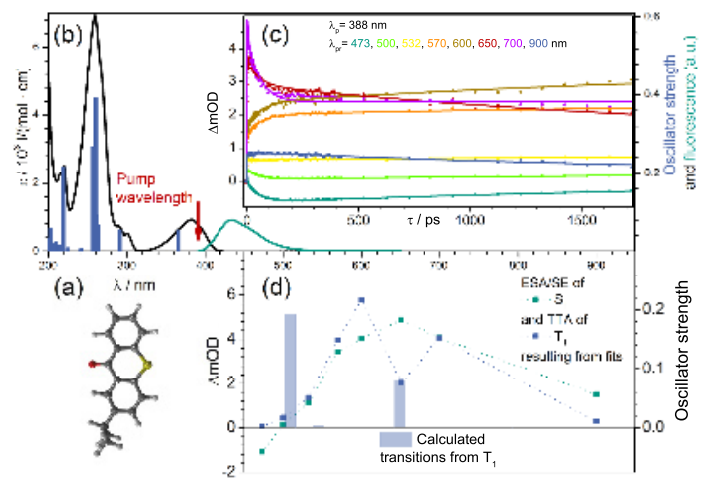

Fig. 3. (a) Structure of ITX, (b) absorption and fluorescence spectra and calculated singlet transitions, (c) transient absorption traces at several $\lambda_{p r}$ with global fit analysis, (d) amplitudes of excited state absorption/stimulated emission (SE/ESA) and triplet-triplet absorption (TTA) together with calculated triplet transitions from $\mathrm{T}_{1}$.

high-performance fluorescence dye: an absorption band, which can be assigned to the $\mathrm{S}_{1} \leftarrow \mathrm{S}_{0}$ transition, with a high extinction coefficient and therefore a strong radiative coupling between $S_{1}$ and $S_{0}$. The coincidence of an also highly efficient ISC channel however reduces the observed fluorescence quantum yield, which makes the molecule ideally suitable for stimulated emission depletion (STED) lithography with a depletion laser at the easily accessible wavelength of $532 \mathrm{~nm}$ (see the excited state absorption (ESA)/stimulated emission (SE) and triplet-triplet absorption (TTA) values extracted from fits in Fig. 2 (d)). Thus, compared to the first two systems, ISC has to compete wih fluorescence for $\mathrm{S}_{1}$ population. The high quantum efficiency is only maintained due to the absence of IC channels.

The intensity of the first absorption band of ITX (see Fig. 3 (b), structure: Fig. 3 (a)), which can be assigned to the $\mathrm{S}_{1} \leftarrow \mathrm{S}_{0}$ transition, is somewhat in between the cases of DETC and Bz. Therefore only weak fluorescence would be expected. Surprisingly, ITX exhibits a fluorescence quantum yield of 15 $\%$ [10]. Thus, any other channel competing for $\mathrm{S}_{1}$ population has to be rather inefficient. Accordingly, the $\mathrm{S}_{1}$ lifetime extracted from the TA traces in Fig. 3 is $2.3 \mathrm{~ns}$. Due to its high $\mathrm{S}_{1}$ lifetime, ITX would be in principle more suitable for STED lithography than DETC. However, as can be seen from the ESA/SE coefficients in Fig. 3 (d), at most depletion wavelengths the ESA probability is higher than $\mathrm{SE}$ and SE depletion is not possible.

In summary, it is demonstrated with the help of four example systems that $\mathrm{S}^{*}$ lifetime cannot serve as a measure for the initiation efficiency of a triplet photoinitiator. In principle, ISC that is the crucial process in the initiation reaction, can compete with IC on an ultrafast time scale as well as with fluorescence on a ns timescale. For an estimation of the ISC quantum efficiency, one has to identify relevant competing processes.

\section{References}

1. F. A. Rueggeberg, Dent. Mater. 27, (2011) 39.

2. A. Biswas, I. S. Bayer, A. S. Biris, T. Wang, E. Dervishi, F. Faupel, Adv. Colloid Interface Sci. 170, (2012) 2.

3. L. Li, R. R. Gattass, E. Gershgoren, H. Hwang, J. Fourkas, Science 324, (2009) 910.

4. J. Fischer, M. Wegener, Laser \& Photon. Rev., (2012) DOI: 10.1002/lpor.201100046.

5. D. Voll, T. Junkers, C. Barner-Kowollik, Macromolecules 44, (2011) 2542.

6. T. J. A. Wolf, D. Voll, C. Barner-Kowollik, A.-N. Unterreiner, Macromolecules 45, (2012) 2257.

7. S. W. Hell, Science 316, (2007) 1153.

8. T. J. A. Wolf, J. Fischer, M. Wegener, A.-N. Unterreiner, Opt. Lett. 36, (2011) 3188.

9. J. Fischer, M. Wegener, Adv. Mater. 24, (2012) OP65.

10. J. Fischer, G. von Freymann, M. Wegener, Adv. Mater. 22, (2010) 3578.

11. R. Ahlrichs, M. Bär, M. Häser, H. Horn, C. Kölmel, Chem. Phys. Lett. 162, (1989) 165. 